\title{
Positive and negative affect mediate the bidirectional relationship between emotional processing and symptom severity and impact in Irritable Bowel Syndrome
}

Alice Sibelli ${ }^{1}$, Prof. Trudie Chalder ${ }^{2}$, Dr. Hazel Everitt ${ }^{3}$, Dr. Joseph Chilcot ${ }^{1}$, Prof. Rona Moss-Morris ${ }^{1 *}$

1. Health Psychology Section, Department of Psychology, Institute of Psychiatry, Psychology and Neuroscience, King's College London, 5th floor Bermondsey Wing, Guy's Hospital Campus, London, SE1 9RT

2. Department of Psychological Medicine, Institute of Psychiatry, Psychology and Neuroscience, King's College London, Weston Education Centre, Cutcombe Road, London SE5 9RJ

3. Primary Care and Population Sciences, University of Southampton, Aldermoor Health Centre, Aldermoor Close, Southampton, SO16 5ST

${ }^{*}$ Correspondence concerning this article should be addressed to Professor Rona Moss-Morris. Address: Health Psychology Section, Department of Psychology, Institute of Psychiatry, Psychology and Neuroscience, King's College London, 5th floor Bermondsey Wing, Guy's Hospital Campus, London, SE1 9RT, UK. Email: rona.moss-morris@kcl.ac.uk 


\section{Abstract}

\section{Introduction}

Individuals with IBS report higher levels of psychological distress compared to healthy controls. Distress has been associated with emotional processing difficulties but studies have not explored how the relationship between distress and emotional processing affects IBS. There is little research on the role of positive affect (PA) in IBS.

\section{Aims}

(a) If difficulties in self-reported emotional processing are associated with affect and IBS measures (i.e., symptom severity, interference in life roles) (b1) If affect mediates the relationship between emotional processing and IBS measures (b2) Alternative model: if affect mediates the relationship between IBS and emotional processing (c) If PA moderates the relationship between distress and IBS.

\section{Methods}

Participants with a confirmed diagnosis of IBS $(n=558)$ completed a questionnaire including measures of emotional processing (i.e., unhelpful beliefs about negative emotions, impoverished emotional experience), distress, PA, and IBS measures. Mediation and moderation analyses were conducted with Maximum Likelihood Estimation.

\section{Results}

Distress and PA mediated or partly mediated the relationship between unhelpful beliefs about negative emotions/impoverished emotional experience and both IBS measures. The alternative models were also valid, suggesting a two-way relationship between emotional processing and IBS through affect. PA did not moderate the relationship between distress and IBS.

\section{Conclusion}

Future interventions in IBS may benefit from not only targeting the management of physical symptoms and their daily impact but also aspects related to the experience of both negative and positive affect, and the acceptance and expression of negative 
Affect as mediator between emotional processing and IBS related measures

emotions. Longitudinal studies are needed to confirm causal relationships within the explored models.

Keywords: Irritable Bowel Syndrome; Emotional processing; Psychological distress; Positive affect; IBS symptom severity; IBS interference in life roles. 
Affect as mediator between emotional processing and IBS related measures

\section{Introduction}

Irritable Bowel Syndrome (IBS) is a chronic gastrointestinal disorder affecting between $10-25 \%$ of individuals in community samples and approximately $11 \%$ of the global population [1, 2]. IBS is not explained by an organic abnormality and it is defined as a functional disorder (i.e. disorder of the gut-brain interaction), diagnosed through symptom-based criteria [3-5].

It is well established that individuals with IBS report higher levels of anxiety and depression compared to healthy controls $[6,7]$ and preliminary findings support the role of anxiety and depression in the development of IBS [8]. Furthermore, anxiety and depression have been associated with difficulties in emotional processing [9-11]. Initial research has found that 1) IBS patients report poorer levels of emotional processing compared to healthy controls [12] and 2) difficulty with identifying and describing feelings has been positively associated with IBS symptom severity [13] and poorer treatment outcome [14]. Interestingly, although previous studies have suggested an effect of psychological distress on IBS symptom severity and quality of life through pain catastrophizing [15, 16], research has not specifically explored how difficulties in processing emotions relate to affect and IBS severity.

Earlier definitions of emotional processing refer to the process through which emotional disturbances are absorbed (i.e. distressed emotional reactions are changed to non-distressed reactions), allowing other experiences and behaviours to continue with minimal disruption $[17,18]$. This initial concept has evolved to incorporate the underlying psychological, psycho-physiological and psychoneurological mechanisms that either promote or impede this change or "absorption". For example, cognitions can be conceived as potential inhibitors of emotional processing, including unhelpful thought patterns, negative appraisals of an event, cognitive biases and catastrophic misinterpretations [10, 18, 19].

Identifying and measuring the different psychological mechanisms underlying emotional processing through self-reported measures can be problematic as it requires some level of awareness of the diminished emotional experience and expression [20]. Research in conditions such as chronic fatigue syndrome (CFS) and depression has focused specifically on the beliefs about the unacceptability of 
experiencing negative emotions and beliefs about the adverse consequences of expressing them [21-24]. This cognitive component of emotional processing allows access to conscious beliefs that disrupt both the experience and expression of emotions.

Initial explorations of beliefs about emotions in IBS have shown that that people with IBS report more negative beliefs about expressing their emotions than both healthy controls and patients with inflammatory bowel disease (IBD) [25, 26]. The relationship between these beliefs and quality of life was serially mediated by emotional suppression and then distress, suggesting that emotional suppression further increases the negative emotions being suppressed in the first place [27]. In line with these findings, written emotional expression has been found to improve IBS symptom severity [28].

Research in other functional disorders such as fibromyalgia replicates these findings [29]; however, the direction of these relationships has not been established yet. It may also be that the experience of psychological distress increases beliefs about the unacceptability of experiencing and expressing the negative emotion [30].

Finally, as noted above, research has extensively studied the association between psychological distress and IBS. Despite findings that positive affect (PA) is linked to better health outcomes in coronary problems and stroke [31, 32], little empirical work has been conducted in IBS [33]. PA has been found to have indirect health benefits by buffering the adverse consequences of perceived stress and pain in conditions such as coronary artery disease and chronic pain [34, 35]. However, PA also appears to have a direct effect on health independent of stress by shaping physiological responses [36]. Thus, exploring the role of PA specifically in IBS could provide valuable information on new ways to treat IBS and improve treatment outcomes.

In summary, whilst the role of psychological distress in IBS has been consistently studied, emotional processing and PA warrant further exploration. Based on previous findings discussed above, difficulties in emotional processing may have a negative impact on IBS related measures through the increase of distress and the reduction of PA. However, it may also be that IBS increases distress and reduces PA, which in 
turn may have a negative impact on processing emotions. Additionally, PA may buffer the relationship between distress and IBS measures. It seems relevant to study unhelpful beliefs about negative emotions as well as the difficulty in identifying emotions as they have been associated with poorer IBS outcomes and treatment responses.

\section{Objectives of the study.}

The current study sought to expand the available knowledge on the areas of research described above. We aimed to investigate:

a) If difficulties in self-reported emotional processing are associated with distress, PA, IBS symptom severity and interference with life roles.

b) How emotional processing is associated with affect and IBS symptom severity and interference with life: (b1) is the relationship between emotional processing and these IBS measures mediated by distress? (b2) is the relationship between emotional processing and these IBS measures mediated by PA? (b3) due to the cross-sectional nature of the data, we tested the reversed models (i.e., IBS measures as predictors, affect variables as mediators, emotional processing measures as dependant variables).

c) If PA moderates the relationship between distress and IBS outcomes.

\section{Methods}

\section{Study population.}

Five hundred and fifty-eight participants were recruited to take part in a randomised controlled trial aimed to assess the clinical and cost effectiveness of CBT in IBS (ACTIB study) [37]. Individuals had to meet the following inclusion criteria:

- Being 18 years and over.

- Having refractory IBS (i.e. fulfilling the ROME III criteria for IBS and reporting ongoing clinically significant symptoms determined by an IBS symptom severity score of 75 or more) [38].

- Having ongoing symptoms of IBS for 12 months or more. 
Affect as mediator between emotional processing and IBS related measures

- Having been offered first-line therapies.

The main exclusion criteria were: unexplained rectal bleeding or weight loss; diagnosis of IBD, coeliac disease, peptic ulcer disease or colorectal carcinoma.

Participants were identified and invited to the study through GP surgeries and secondary care clinics in two regions (Southampton and London). Those individuals responding to the invitation letter were contacted by the research team and completed a screening telephone interview covering inclusion/exclusion questions as well as the ROME III criteria. Eligible participants consented online through the study website and agreed to have routine blood tests. Participants with normal blood test results (i.e. full blood count, tissue transglutaminase antibodies and C-reactive protein) completed the baseline questionnaires online (see study protocol for further details on the recruitment process and ethics approvals) [37]. The current study was a cross-sectional analysis of these baseline data.

\section{Measures.}

\section{Emotional processing and affect measures:}

Beliefs about Emotions Scale (BES).

The BES [24] is a 12-item scale that measures beliefs about the unacceptability of experiencing and expressing negative emotions. Items are answered on a 7-point Likert scale ( 0 to 6$)$ and higher scores indicate stronger negative beliefs about emotions. The BES has one factor and it showed very good reliability in our study (Cronbach's $\alpha=0.91$ ).

Impoverished Emotional Experience (IEE) sub-scale.

The IEE factor of the refined Emotional Processing Scale (EPS-25) [19] captures some aspects of the alexithymia construct. Specifically, it is focused on the difficulty in differentiating between feeling ill or emotional and the dissociation from the emotional experience. IEE correlates most highly with the other sub-scales and it had high internal consistency (Cronbach's $\alpha=0.88$ ). This sub-scale is composed of 5 items answered on a 10-point Likert scale (0 to 9). Higher scores correspond to poorer emotional experience. 
Hospital Anxiety and Depression Scale (HADS).

The HADS [39] is a 14-item, valid and reliable self-report instrument for detecting anxiety and depression in individuals with medical conditions. The scale is answered using a 4-point Likert scale ( 0 to 3 ) and higher scores indicate higher levels of distress. We conducted Confirmatory Factor Analysis in Mplus [40] with our data and concluded that a bi-factor model, consisting of a general distress factor and two smaller factors (anxiety and depression), most appropriately fitted the data. Seventythree per cent of the explained variance (omega-h) was accounted for by the general factor, which makes it sufficiently unidimensional to warrant use of the total score. In line with previous research [41, 42], it seems appropriate to use a total HADS score as a reliable measure of general psychological distress rather than two separate precise measures of anxiety and depression (Cronbach's $\alpha=0.87$ ).

Positive and Negative Affect Schedule (PANAS).

The PANAS [43] is composed of two independent factors measuring positive and negative affect. Only the PA scale was used in the current study. This subscale has 10 items answered on a 5-point Likert scale (1 to 5). Higher scores indicate higher levels of PA. The current study used the timeframe "during the past week" and the sub-scale showed a Cronbach's $\alpha$ of 0.92 .

\section{Dependent variables/outcomes:}

The IBS Symptom Severity Score (IBS-SSS).

The IBS-SSS [38] is a 5-item self-administered instrument measuring severity and duration of abdominal pain, abdominal distension, bowel habits and quality of life. The scale showed a Cronbach's $\alpha$ of 0.69 . The maximum score is 500 and higher scores indicate worse symptom severity.

The Work and Social Adjustment Scale (WSAS).

The WSAS [44] is a 5-item questionnaire measuring the effect of IBS on patients' ability to work and manage a home, participate in social and private leisure activities, and maintain relationships. Based on the World Health Organisation definition of function and disability, the WSAS is a measure of patient restrictions or interference in life situations [45]. Items are answered on a 9-Likert scale (0 to 8) and higher 
scores correspond to higher patient restrictions. It showed good reliability (Cronbach's $\alpha=0.85$ )

\section{Statistical methods.}

The Cronbach's a for each scale was calculated using IBM SPSS Statistics 21. Bivariate Pearson correlations to explore the strength and direction of the associations between the emotional processing variables, affect and IBS related measures were conducted using IBM SPSS Statistics 21 [46]. $r$ coefficient and $p$ value for the correlation coefficients are reported. Mediation and moderation analyses were conducted using MPlus 7.4 [40] with Maximum Likelihood Estimation $(\mathrm{ML})$. Estimates and $p$ values are reported for the standardised path coefficients. Bootstrap (10,000 samples) and bias corrected (BCa) $95 \% \mathrm{Cl}$ of the standardised indirect effect were calculated taking into account possible non-normality of the effect distributions [47]. We controlled for age, gender, marital status and ethnic background. All path models tested had acceptable fit as determined by standard fit criteria (i.e. CFI, TLI and RMSEA) [48]. There were no missing data.

\section{Results}

\section{Demographic and clinical variables.}

Our sample ( $\mathrm{N}=558)$ was predominantly composed of women $(76 \%)$ from a white ethnic background. Approximately $60 \%$ of participants were married or living with a partner and $60 \%$ had completed a degree or postgraduate studies (see Table 1 for demographic characteristics of the sample). On average, participants had moderate symptoms of IBS (see Table 2 for clinical characteristics). 
Table 1

Demographic characteristics (categorical) $(n=558)$

\begin{tabular}{lcc}
\hline \multicolumn{1}{c}{ Variable } & Number & Percentage \\
\hline Gender & & \\
Male & 135 & 24.19 \\
Female & 423 & 75.81 \\
\hline Ethnicity & 507 & \\
White & 11 & 90.86 \\
Mixed / multiple ethnicities & 2 & 1.97 \\
Asian / Asian British & 5 & 0.36 \\
Other group & 33 & 0.90 \\
Missing & 149 & 5.91 \\
\hline Marital status & 263 & 26.70 \\
Single & 98 & 47.13 \\
Married & 11 & 17.56 \\
Living with partner & 27 & 1.97 \\
Separated & 10 & 4.84 \\
Divorced & & 1.79 \\
Widowed & 22 & 3.94 \\
\hline Educational level & 112 & 20.07 \\
No formal & & \\
GCSE/O level or & 83 & 14.87 \\
equivalent & 190 & 34.05 \\
A level or equivalent & 109 & 19.53 \\
Degree & 42 & 7.53 \\
Postgraduate & & \\
other & &
\end{tabular}


Affect as mediator between emotional processing and IBS related measures

Table 2

Descriptive statistics of clinical variables $(n=558)$

\begin{tabular}{|c|c|c|c|c|}
\hline Variable & Mean & SD & Median & Range \\
\hline $\begin{array}{l}\text { Beliefs about } \\
\text { emotions } \\
\text { (scores from 0- } \\
72 \text { ) }\end{array}$ & 39.34 & 14.05 & & \\
\hline $\begin{array}{l}\text { Impoverished } \\
\text { emotional } \\
\text { experience } \\
\text { (scores from 0- } \\
45 \text { ) }\end{array}$ & & & 17.00 & $0-45$ \\
\hline $\begin{array}{l}\text { Positive affect } \\
\text { (scores from 1- } \\
50)\end{array}$ & 30.20 & 8.80 & & \\
\hline $\begin{array}{c}\text { HADS } \\
\text { anxiety } \\
\text { (scores from 0- } \\
21 \text { ) }\end{array}$ & 10.72 & 4.19 & 11 & $1-21$ \\
\hline $\begin{array}{c}\text { HADS } \\
\text { depression } \\
\text { (scores from 0- } \\
21)\end{array}$ & 5.65 & 3.66 & 5 & $0-19$ \\
\hline $\begin{array}{l}\text { HADS distress } \\
\text { (scores from 0- } \\
42 \text { ) }\end{array}$ & 16.38 & 6.88 & & \\
\hline $\begin{array}{c}\text { Symptom } \\
\text { severity score } \\
\text { (scores from 0- } \\
500)\end{array}$ & 264.98 & 95.48 & & \\
\hline $\begin{array}{l}\text { WSAS score* } \\
\text { (scores from 0- } \\
40 \text { ) }\end{array}$ & & & 11 & $0-40$ \\
\hline $\begin{array}{l}\text { Age at } \\
\text { randomisation }\end{array}$ & & & 42.50 & $18.8-81$ \\
\hline $\begin{array}{l}\text { Years from } \\
\text { start of } \\
\text { symptoms to } \\
\text { diagnosis }\end{array}$ & & & 3 & $0-47$ \\
\hline
\end{tabular}


Affect as mediator between emotional processing and IBS related measures

Years from

7.38

$0.31-64.57$

diagnosis to

randomisation)

Note. Mean and standard deviation are given for normally distributed variables. Median and range are given for non-normally distributed variables, and for both HADS sub-scales.

*WSAS: Work and Social Adjustment Scale.

Female participants reported significant higher levels of anxiety compared to male participants. Participants who were single, separated, divorced or widowed (group 1) reported higher levels of impoverished emotional experience, anxiety, overall distress, IBS symptom severity and interference with life roles compared to participants living with partner/married (group 2). In terms of ethnicity, participants from a white background reported lower levels of IBS interference with life, depression and better emotional processing (in relation to both measures) compared to participants from all other backgrounds (see Appendix 1 for detailed results). 


\section{Correlations.}

Table 3 presents the associations between the proposed predictor, mediator and outcome variables. We found small positive associations between both emotional processing variables and IBS symptom severity and interference with life.

Furthermore, beliefs about emotions and emotional impoverished experience were correlated with the proposed mediators; positively with distress and negatively with PA. Overall, both emotional processing variables showed stronger associations with affect than with IBS measures. Distress correlated positively with IBS symptom severity and interference with life whilst PA showed negative associations with them.

Table 3

Correlations between emotional processing variables, $P A$, distress and IBS related measures.

\begin{tabular}{|c|c|c|c|c|c|c|c|}
\hline & BES & IEE & IBS-SSS & WSAS & HADS-A & HADS-D & $\begin{array}{c}\text { HADS } \\
\text { distress }\end{array}$ \\
\hline IEE & $0.48^{* *}$ & - & & & & & \\
\hline IBS-SSS & $0.14^{\star *}$ & $0.16^{\star *}$ & - & & & & \\
\hline WSAS & $0.25^{\star *}$ & $0.33^{* *}$ & $0.49^{\star *}$ & - & & & \\
\hline HADS-A & $0.45^{\star *}$ & $0.52^{\star *}$ & $0.24^{\star *}$ & $0.30^{* *}$ & - & & \\
\hline HADS-D & $0.45^{\star \star}$ & $0.56^{\star *}$ & $0.24^{\star \star}$ & $0.43^{\star *}$ & $0.53^{\star *}$ & - & \\
\hline $\begin{array}{c}\text { HADS } \\
\text { distress }\end{array}$ & $0.51^{* *}$ & $0.62^{\star \star}$ & $0.27^{\star *}$ & $0.41^{* *}$ & $0.89^{* *}$ & $0.86^{\star *}$ & - \\
\hline PA & $-0.35^{\star *}$ & $-0.49^{* *}$ & $-0.21^{* *}$ & $-0.31^{* *}$ & $-0.43^{* *}$ & $-0.68^{* *}$ & $-0.62^{\star *}$ \\
\hline
\end{tabular}




\section{Mediation and moderation analyses.}

\section{Distress as mediator between emotional processing and IBS related measures.}

IBS interference with life roles

As predicted, distress mediated the relationship between beliefs about emotions and IBS interference with life roles (see Figure 1). Indirect effect: $\beta=0.19, \mathrm{BCa} 95 \% \mathrm{Cl}$ [0.14-0.24], $p<0.001$. With distress in the model, the direct effect of beliefs about emotions on IBS interference was not significant. With the present model, approximately $25.9 \%$ of the variability in distress and $22.3 \%$ of the variability in IBS interference was explained; $83 \%$ of the total effect was accounted for by distress.

Distress also mediated the relationship between impoverished emotional experience and IBS interference (see Figure 1). Indirect effect: $\beta=0.21$, BCa 95\% [0.14-0.27], $p<0.001$. The direct effect was not significant. With this model, $38.1 \%$ of the variability in distress and $23.3 \%$ variability in IBS interference was explained; $70 \%$ of the total effect is accounted for by the mediator.

\section{Proposed model}
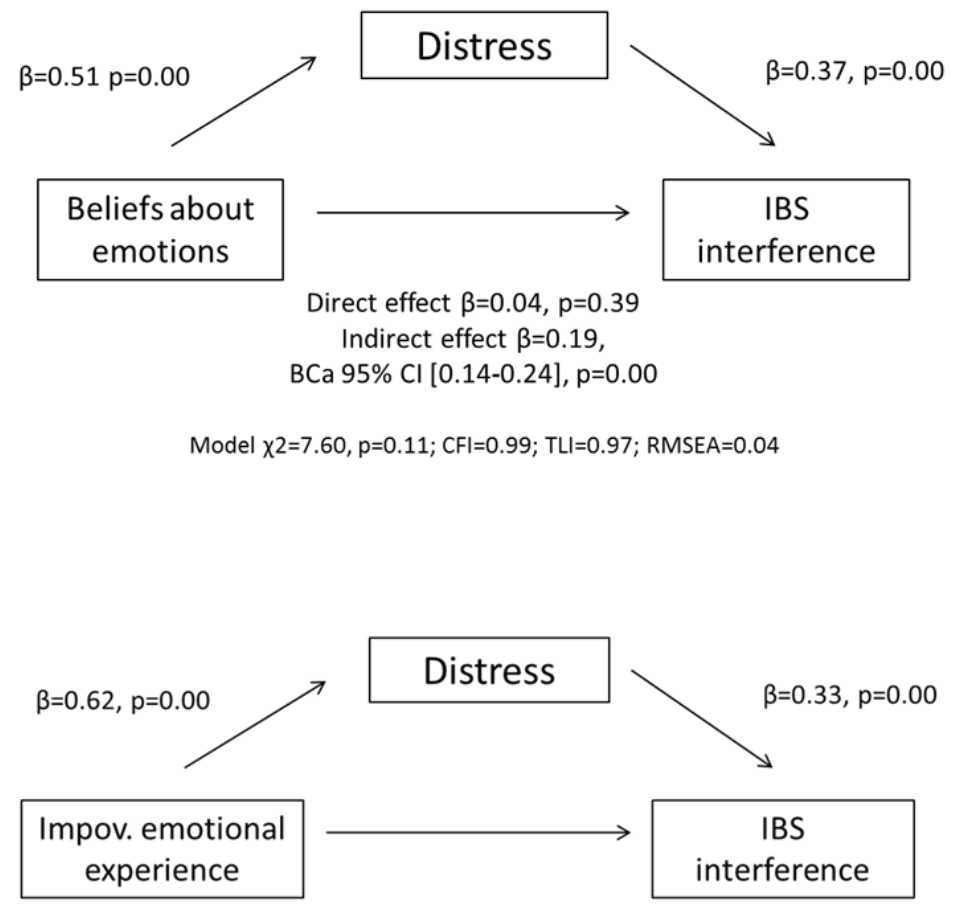

Direct effect $\beta=0.09, p=0.07$

Indirect effect $\beta=0.21$,

BCa $95 \% \mathrm{Cl}[0.14-0.27], p=0.00$

Model $\chi 2=7.45, \mathrm{p}=0.11 ; \mathrm{CFI}=0.99 \mathrm{TLI}=0.98 \mathrm{RMSEA}=0.04$ 
Figure 1

Diagrams showing the results of distress as mediator between emotional processing variables (i.e. beliefs about emotions and impoverished emotional experience) and IBS interference with life roles.

\section{IBS symptom severity}

As hypothesised, distress mediated the relationship between negative beliefs about emotions and IBS symptom severity (see Figure 2). Indirect effect: $\beta=0.13$, BCa $95 \%$ $\mathrm{Cl}[0.08-0.19], p<0.001$. The direct effect of beliefs about emotions on symptom severity was not significant. With the present model, $25.9 \%$ of the variability in distress and $10.4 \%$ of the variability in symptom severity was explained; $93 \%$ of the total effect was accounted for by the mediated path.

Distress also mediated the relationship between impoverished emotional experience and symptom severity (see Figure 2). The indirect effect was significant $(\beta=0.17$, $\mathrm{BCa} 95 \% \mathrm{Cl}[0.10-0.24], \mathrm{p}<0.001)$, whilst the direct effect was not. With this model, $38.1 \%$ of the variability in distress and $10.5 \%$ of the variability in symptom severity was explained; $90 \%$ of the total effect was accounted for by the mediator. 
Proposed model
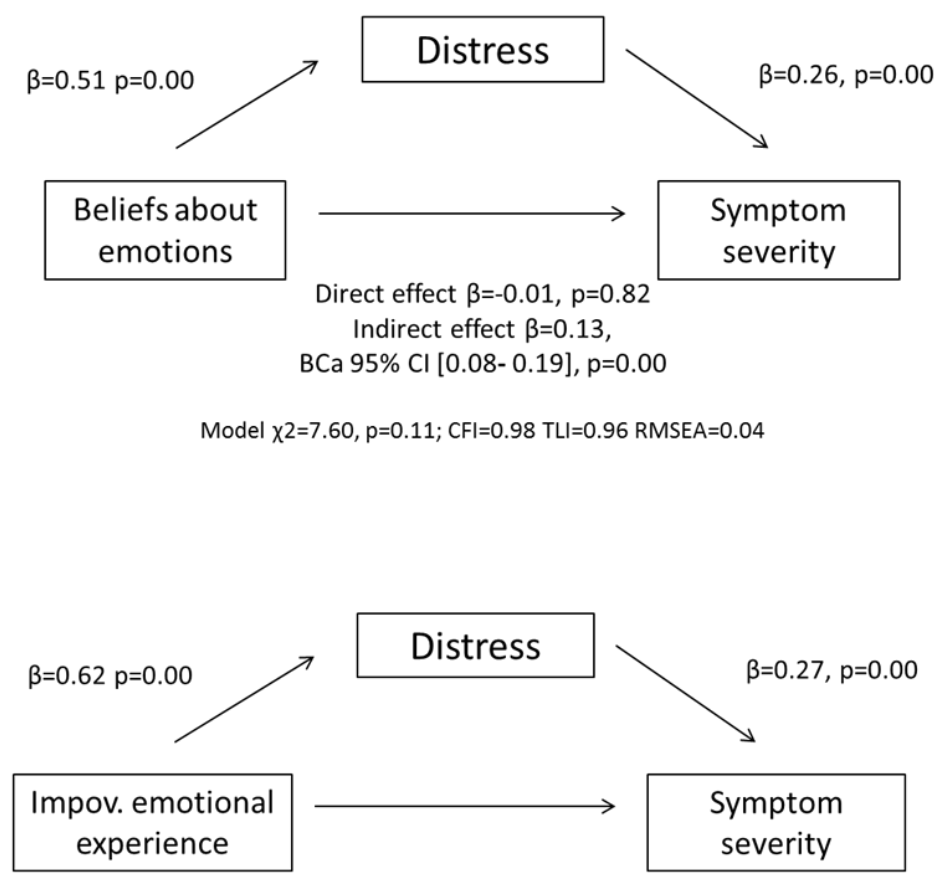

Direct effect $\beta=-0.02, p=0.71$

Indirect effect $\beta=0.17$,

$\mathrm{BCa} 95 \% \mathrm{Cl}[0.10-0.24], \mathrm{p}=0.00$

Model $\chi 2=7.45, \mathrm{p}=0.11 ; \mathrm{CFI}=0.99 \mathrm{TLI}=0.97 \mathrm{RMSEA}=0.04$

Figure 2

Diagrams showing the results of distress as mediator between emotional processing variables (i.e., beliefs about emotions and impoverished emotional experience) and IBS symptom severity.

\section{Positive affect as mediator between emotional processing and IBS related measures.}

IBS interference with life roles

Positive affect partially mediated the relationship between negative beliefs about emotions and IBS interference with life roles (see Figure 3). Indirect effect: $\beta=0.09$, $\mathrm{BCa} 95 \% \mathrm{Cl}[0.05-0.13], p<0.001$. The direct effect of beliefs about emotions was significant: $\beta=0.14, p<0.001$. In this model, $39 \%$ of the total effect was accounted for by the indirect effect. 
Positive affect also partially mediated the relationship between impoverished emotional experience and IBS interference (see Figure 3). Indirect effect: $\beta=0.10$, $\mathrm{BCa} 95 \% \mathrm{Cl}$ [0.04-0.12], $\mathrm{p}<0.001$. The direct effect of impoverished emotional experience was significant: $\beta=0.20, p<0.001$. In this model, $33 \%$ of the total effect was accounted for by the indirect effect.

\section{Proposed model}
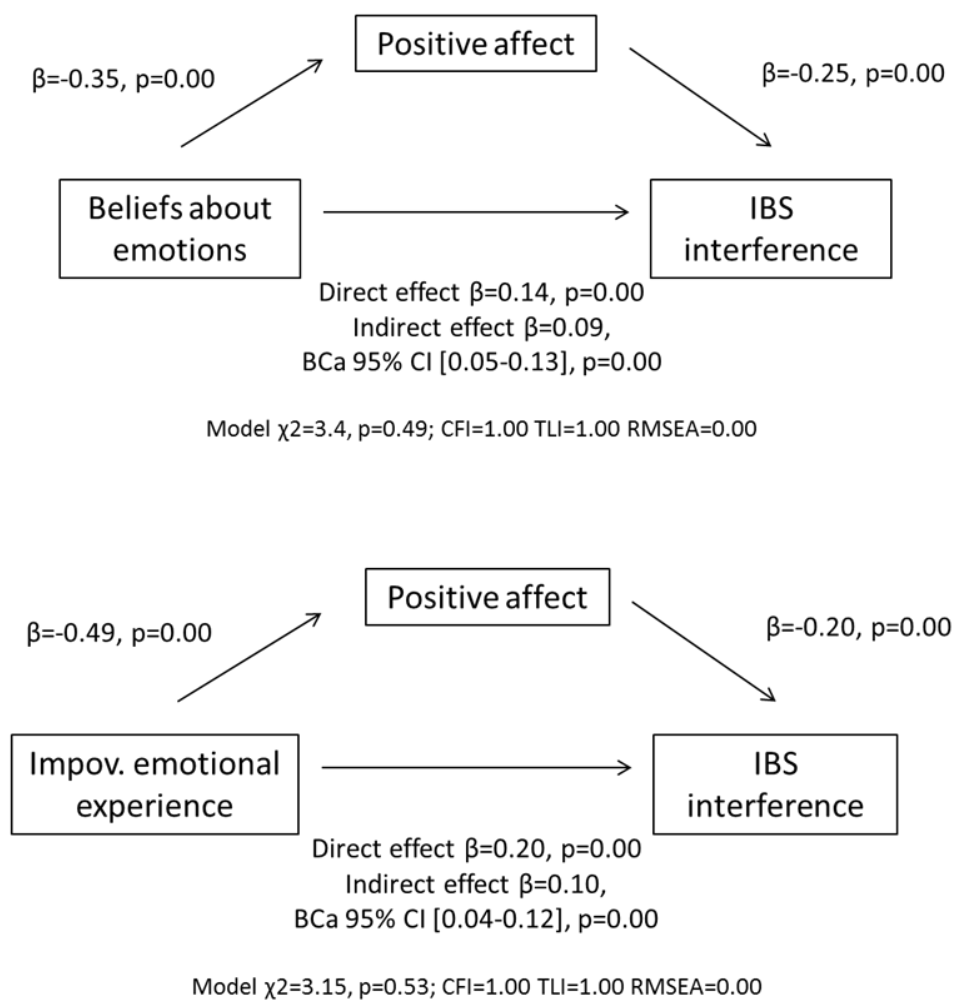

Figure 3

Diagrams showing the results of positive affect as mediator between emotional processing variables (i.e., beliefs about emotions and impoverished emotional experience) and IBS interference with life roles.

\section{IBS symptom severity}

As predicted, positive affect mediated the relationship between negative beliefs about emotions and IBS symptom severity (see Figure 4). Indirect effect: $\beta=0.07$, $\mathrm{BCa} 95 \% \mathrm{Cl}$ [0.26-0.73], $\mathrm{p}<0.001$. The direct effect of beliefs about emotions was not significant. With the present model, $12.1 \%$ of the variability in PA and $9.8 \%$ of the 
variability in symptom severity was explained; $58 \%$ of the total effect was accounted for by the mediator.

PA also mediated the relationship between impoverished emotional experience and IBS symptom severity (see Figure 4). Indirect effect: $\beta=0.10, \mathrm{BCa} 95 \% \mathrm{Cl}[0.05-$ $0.15], p<0.001$. The direct effect was not significant. With this model, $24.4 \%$ of the variability in PA and $9.8 \%$ of the variability in symptom severity was explained; $67 \%$ of the total effect was accounted for by the mediated path.

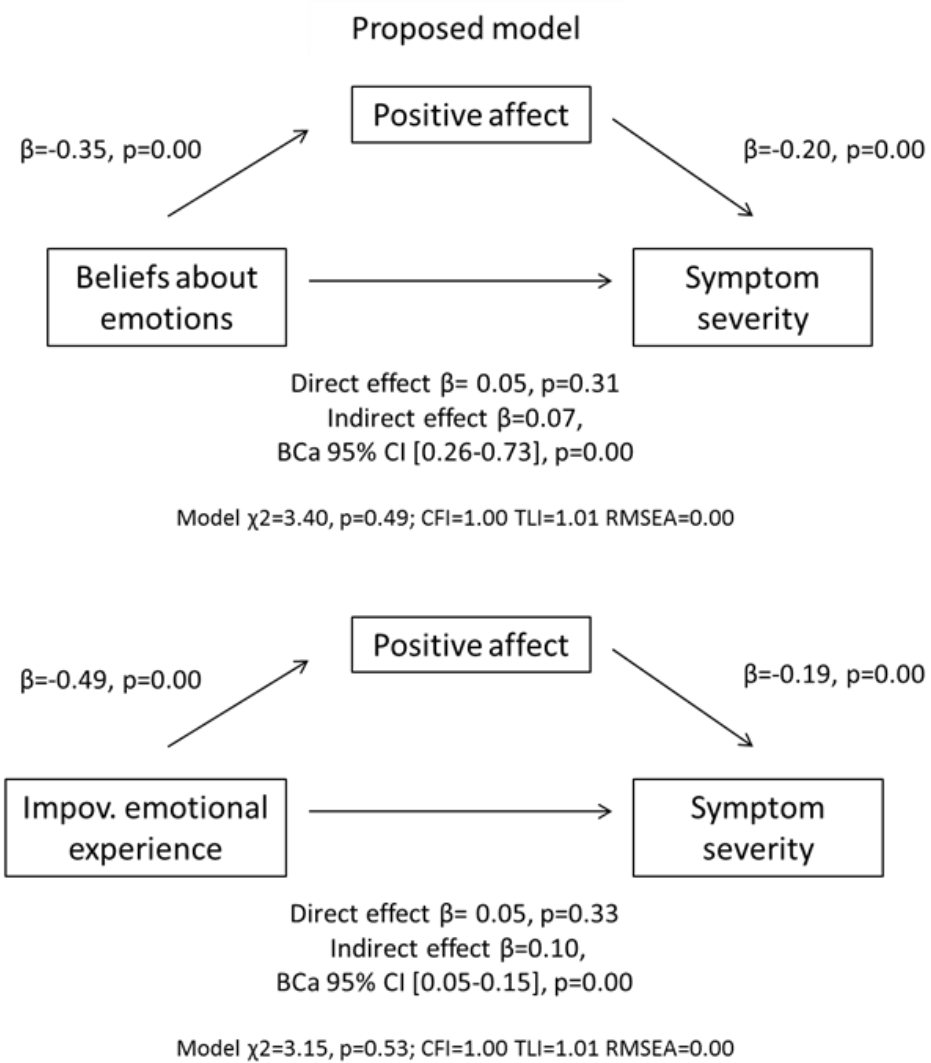

Figure 4

Diagrams showing the results of positive affect as mediator between emotional processing variables (i.e., beliefs about emotions and impoverished emotional experience) and IBS symptom severity. 


\section{Testing the alternative models.}

Due to the fact that the directional hypotheses were based on cross-sectional data, we also ran the reversed models (i.e., IBS measures as predictors, affect variables as mediators, emotional processing measures as dependant variables). Overall, all alternate models showed similar effects to the ones we proposed (see Appendices 2 and 3 for detailed results).

\section{Positive affect as moderator between distress and IBS related measures.}

Contrary to our hypothesis, PA did not moderate the relationship between overall distress and IBS interference in life roles as the interaction effect was not significant: $\beta=-0.04, \mathrm{BCa} 95 \% \mathrm{Cl}[-0.26-0.17], p=0.73$. Similar results were found for IBS symptom severity: $\beta=0.07, \mathrm{BCa} 95 \% \mathrm{Cl}[-0.14-0.26], p=0.50$.

\section{Discussion}

\section{Summary of results.}

To our knowledge, this is the first study to explore the associations between emotional processing variables, psychological distress, PA and IBS symptom severity and interference in life roles. We found that negative beliefs about emotions and impoverished emotional experience showed small significant positive associations with distress, IBS symptoms and interference. In contrast, both beliefs about emotions and impoverished emotional experience showed small significant negative associations with PA. Overall, the emotional processing variables were more strongly associated with affect than IBS symptom severity. The correlations between the emotional processing variables and IBS interference were also larger than the associations with IBS symptom severity.

In terms of demographic variables, participants from a white background had lower levels of difficulties in emotional processing compared to those from all other backgrounds. This seems to be in line with previous studies conducted with both clinical and non-clinical samples, showing that individuals from a white/Caucasian background present less difficulties in emotional processing compared to participants from other ethnicities $[49,50]$, perhaps due to social desirability [51]. This study had 
low numbers of participants from non-white ethnicities and future research should focus on the associations between difficulties in emotional processing in individuals with IBS from different ethnic and cultural backgrounds to understand more clearly how these variables may influence IBS outcomes and response to treatments.

In line with hypotheses, mediation analyses found that distress fully mediated the relationship between both emotional processing variables and IBS measures. Similarly, PA fully mediated the relationship between emotional processing and IBS symptom severity and partially mediated the relationship between emotional processing and IBS interference in life roles. This suggests that negative beliefs about the acceptability of emotions and impoverished emotional experience are associated with higher levels of distress and lower levels of PA, which in turn are associated with increased symptom severity and IBS impact on life roles.

Interestingly, the mediation analyses of the alternate models also showed significant results, suggesting that higher levels of IBS symptom severity and interference with life are associated with increased psychological distress and lowered PA, which in turn are associated with difficulties in emotional processing. Thus, the mediation results supported both the proposed and reversed models. Finally, contrary to our hypothesis, PA did not moderate the relationship between distress and IBS measures.

\section{Proposed models.}

Our findings suggested that the selected emotional processing variables have an impact on IBS measures through generated greater distress and dampening the experience of PA. Others have shown that suppression of emotions was associated with physiological and physical responses usually triggered by distress: increases in sympathetic nervous system activity, heightened cortisol reactivity in response to stressors, higher levels of inflammation, slower gastric emptying and more intense fatigue [23, 36, 52-56]. These alterations may affect the normal functioning of the nervous, immune and endocrine systems and can increase the strength/length of peristaltic movements, changing bowel patterns and enhancing the sensation of pain [57]. 
Our results also suggested that unhelpful beliefs about emotions and impoverished emotional experience were associated with higher levels of IBS interference in social and work functioning. Cognitive behavioural models of medically unexplained symptoms posit that negative beliefs about emotions lead people to bottle-up emotions and to avoid asking for help, potentially affecting the quality of the relationships and/or increasing the sense of isolation [21, 25]. This may then increase psychological distress, which can further have a negative effect on cognitive functioning and on the ability to relax or enjoy leisure activities [58].

Beliefs about the unacceptability of experiencing and expressing negative emotions can be addressed and reframed during therapy [59], eliciting conscious answers about suppressing behaviours that can sometimes be automatic for the individual. CBT and mindfulness-based CBT for CFS have been associated with a decrease in unhelpful beliefs about emotions [24, 60]. Similarly, a study found that expressive writing significantly decreased IBS symptom severity and improved IBS related unhelpful cognitions [28]. Thus, addressing beliefs about emotions and promoting emotional expression will likely have a positive impact on IBS outcomes but future controlled studies are needed to confirm this.

Our results also proposed that feeling detached from the emotional experience, not being able to label emotions or to differentiate between feelings or physical sensations, increased distress. Taking this into account as well as previous research on the role of alexithymia in IBS outcomes [61], interventions in IBS may benefit from mindfulness and emotional awareness techniques. Considering that CBT-based interventions have been found successful in improving IBS treatment outcomes [6264], adding emotional awareness techniques [65] to CBT protocols may further improve their efficacy.

One of the most novel findings of our study is that emotional processing variables also seemed to influence IBS related measures, particularly IBS symptom severity, through PA. Addressing unhelpful beliefs about the acceptability of negative emotions and improving emotional awareness may increase the levels of PA, which in turn may have a positive effect on symptom severity and partially on IBS interference with life roles. 
These results suggest that improving levels of PA in participants with emotional processing difficulties may be an additional way to enhance the efficacy of treatments for IBS. PA may have direct effects on disease progression and outcomes via: 1) behavioural changes (e.g. improving socialisation, promoting health practices such as sleep hygiene, adherence to medications) and 2) physiological changes (e.g. endogenous opioids; lower levels of cortisol; norepinephrine and the pro-inflammatory cytokine IL-6) [66-68]. In fact, research shows that PA has a positive effect on patient motivation, readiness to change and self-efficacy in chronic pain [69]. Thus, interventions targeting PA may play a particularly important role in promoting self-management in individuals with chronic conditions.

Interestingly, our results showed that PA did not buffer the relationship between distress and IBS measures. This may be due to the fact that the PANAS measures high-activation PA (e.g. excitement, enthusiasm) but it does not include lowactivation PA terms (e.g. calm, relaxed) [66]. Whorwell and colleagues found that relaxation decreased colonic motility whilst excitement increased it (not as much as anger) [70]. Providing opportunities to experience low activation PA may be an important component of therapy. Additionally, it could be that the role of PA as a stress buffer is different between healthy individuals and individuals with a chronic condition, or even between different chronic conditions. Further studies are needed to confirm or refute these initial findings and to explore the different effects that both types of PA have on IBS.

Overall, it seems relevant to highlight the potential role that regulating both positive and negative emotions can have on improving IBS symptoms and promoting patient involvement in life. Appleton and colleagues (2013) found that reappraisal (i.e. changing cognitions to influence an emotional response) was associated with a reduced risk of having levels of inflammation linked to cardiovascular disease. Furthermore, reappraisal seems to increase positive emotions, decrease negative emotions and enhance memory in specific circumstances [71, 72]. Thus, future studies should explore the helpful and unhelpful emotion regulation strategies relevant to IBS patients and how these can be addressed in interventions. 
Affect as mediator between emotional processing and IBS related measures

\section{Alternative models.}

A limitation of the mediation analyses conducted to explore the proposed models was the use of cross-sectional data. Therefore, we tested the alternate models, which also seemed to be valid: IBS related measures were associated with increased distress and lowered PA, which in turn were associated with difficulties in emotional processing.

The results of the present study suggest a two-way relationship between emotional processing and IBS related measures through affect, raising two important points: 1) it seems crucial to test both types of models with longitudinal data in order to establish clear causal pathways and 2) the alternate models propose further potential mechanistic pathways, highlighting important clinical implications.

The biopsychosocial model of IBS maintains that symptom onset is then followed by unhelpful coping behaviours and illness cognitions, which can perpetuate symptoms and increase psychological distress [73-75]. This conceptualisation of IBS provides support for the relationships found between IBS related variables and distress in our alternate models, highlighting the psycho-emotional effects of IBS [76, 77]. However, research does not provide definite evidence on how affect can impact on unhelpful beliefs about emotions and impoverished emotional experience (dependent variables in the reversed models). An increase in psychological distress may promote and/or reinforce unhelpful beliefs about experiencing and expressing negative emotions [30]. Furthermore, the experience of distress can trigger the use of emotional avoidance as an unhelpful coping strategy [78], potentially promoting detachment from the emotional experience.

As stated above, PA can induce behavioural changes [31, 68], such as socialisation and expression of emotions, diminishing beliefs about the unacceptability of negative emotions. PA has also been associated with diverse cognitive changes [32], which may improve the awareness of the emotional experience. However, these pathways need further exploration in future studies.

Theoretical models seem to provide a stronger support for the proposed models as they tend to conceive psychological distress and low PA a result of emotion regulation problems [79]. Nonetheless, this does not deny the effect that higher 
levels of distress and lower PA may have, in turn, on emotional processing difficulties.

\section{Strengths and limitations.}

To our knowledge, this is the first study to measure both positive and negative affect and explore their relationships with emotional processing variables and IBS symptom severity and interference in life. The measures used to capture emotional processing difficulties are potentially modifiable elements in therapy, which makes our findings clinically relevant. The large sample size is a further strength.

The main limitation of this study is that we cannot infer causal relationships between the variables as this is a cross-sectional analysis. Thus, we cannot exclude that all measures are actually correlated due to construct, content or method overlap, rather than presenting bidirectional mechanisms. For example, they could share variance with some personality features, such as neuroticism, which could lead to the pattern of obtained relationships. Other limitations include: the indirect effects are not strong and may be significant due the large sample size; participants were recruited in the context of a trial offering CBT, which may make them systematically different to other IBS patients; the PANAS uses a measure of hyperarousal PA and it excludes lowactivated moods.

\section{Future research.}

Firstly, it seems critical to conduct longitudinal studies to analyse causal relationships between emotional processing variables, affect and IBS outcomes to confirm and expand the results of the current cross-sectional study and to investigate the temporal direction of these relationships.

Secondly, Desteno and colleagues (2013) pointed out that future research in this area should focus on the role that specific negative and positive emotions have on physiology, cognitions and behaviours, which would promote further insight into the mechanisms linking emotions, health and disease. Furthermore, the changes that emotions trigger on different systems are likely to be associated with the intensity, duration and frequency of the emotion (not only to the type); these aspects are not usually investigated enough in studies. 
Finally, using different measures of PA and psychological distress (e.g. self-reported questionnaires, physiological measurements, biological markers) can help to elucidate the complex relationships between affect, emotional processing difficulties, emotion regulation techniques and IBS.

\section{Conclusion}

Taking into account both the proposed and alternative models, it seems relevant to target both positive and negative affect in IBS interventions. The proposed models suggest an innovative way to improve IBS symptom severity and IBS interference with life roles through changes in affect: addressing beliefs about the unacceptability of experiencing and expressing negative emotions, and promoting emotional expression and awareness. The alternate models propose that diminishing IBS symptom severity and interference with life may improve emotional processing difficulties through changes in distress and PA.

Considering the potential bi-directional relationships between IBS, affect and emotional processing suggested by these models, the findings of our study highlight the importance of implementing tailored interventions for individuals with IBS based on multi-assessments, targeting not only the management of physical symptoms and their impact on life roles but also aspects related to the emotional experience, expression and coping. 


\section{References}

1. Canavan, C., J. West, and T. Card, The epidemiology of irritable bowel syndrome. Clin Epidemiol, 2014. 6: p. 71-80.

2. Lovell, R.M. and A.C. Ford, Global prevalence of and risk factors for irritable bowel syndrome: a meta-analysis. Clin Gastroenterol Hepatol, 2012. 10(7): p. 712-721 e4.

3. Drossman, D.A., The functional gastrointestinal disorders and the Rome III process. Gastroenterology, 2006. 130(5): p. 1377-90.

4. Simren, M., O.S. Palsson, and W.E. Whitehead, Update on Rome IV Criteria for Colorectal Disorders: Implications for Clinical Practice. Curr Gastroenterol Rep, 2017. 19(4): p. 15.

5. Schmulson, M.J. and D.A. Drossman, What Is New in Rome IV. Journal of Neurogastroenterology and Motility, 2017. 23(2): p. 151-163.

6. Henningsen, P., T. Zimmermann, and H. Sattel, Medically unexplained physical symptoms, anxiety, and depression: a meta-analytic review. Psychosom Med, 2003. 65(4): p. 528-33.

7. Fond, G., et al., Anxiety and depression comorbidities in irritable bowel syndrome (IBS): a systematic review and meta-analysis. Eur Arch Psychiatry Clin Neurosci, 2014. 264(8): p. 65160.

8. Sibelli, A., et al., A systematic review with meta-analysis of the role of anxiety and depression in irritable bowel syndrome onset. Psychol Med, 2016. 46(15): p. 3065-3080.

9. Joormann, J. and I.H. Gotlib, Emotion regulation in depression: relation to cognitive inhibition. Cogn Emot, 2010. 24(2): p. 281-98.

10. Baker, R., et al., Emotional processing and panic. Behav Res Ther, 2004. 42(11): p. 1271-87.

11. Martin, R.C. and E.R. Dahlen, Cognitive emotion regulation in the prediction of depression, anxiety, stress, and anger. Personality and Individual Differences, 2005. 39(7): p. 1249-1260.

12. Phillips, K., B.J. Wright, and S. Kent, Psychosocial predictors of irritable bowel syndrome diagnosis and symptom severity. J Psychosom Res, 2013. 75(5): p. 467-74.

13. Porcelli, P., M. De Carne, and G. Leandro, Alexithymia and gastrointestinal-specific anxiety in moderate to severe irritable bowel syndrome. Comprehensive Psychiatry, 2014. 55(7): p. 1647-1653.

14. Porcelli, P., M. De Carne, and G. Leandro, The role of alexithymia and gastrointestinalspecific anxiety as predictors of treatment outcome in irritable bowel syndrome.

Comprehensive Psychiatry, 2017. 73: p. 127-135.

15. Sherwin, L.B., E. Leary, and W.A. Henderson, The association of catastrophizing with qualityof-life outcomes in patients with irritable bowel syndrome. Qual Life Res, 2017. 26(8): p. 2161-2170.

16. van Tilburg, M.A., O.S. Palsson, and W.E. Whitehead, Which psychological factors exacerbate irritable bowel syndrome? Development of a comprehensive model. J Psychosom Res, 2013. 74(6): p. 486-92.

17. Rachman, S., Emotional Processing. Behaviour Research and Therapy, 1980. 18(1): p. 51-60.

18. Rachman, S., Emotional processing, with special reference to post-traumatic stress disorder. International Review of Psychiatry, 2001. 13(3): p. 164-171.

19. Baker, R., et al., The Emotional Processing Scale: scale refinement and abridgement (EPS-25). J Psychosom Res, 2010. 68(1): p. 83-8.

20. Vanheule, S., Challenges for alexithymia research: a commentary on "The construct of alexithymia: associations with defense mechanisms". J Clin Psychol, 2008. 64(3): p. 332-7.

21. Surawy, C., et al., Chronic fatigue syndrome: a cognitive approach. Behav Res Ther, 1995. 33(5): p. 535-44.

22. Cramer, K.M., M.D. Gallant, and M.W. Langlois, Self-silencing and depression in women and men: Comparative structural equation models. Personality and Individual Differences, 2005. 39(3): p. 581-592. 
23. Rimes, K.A., et al., Emotional suppression in chronic fatigue syndrome: Experimental study. Health Psychol, 2016. 35(9): p. 979-86.

24. Rimes, K.A. and T. Chalder, The Beliefs about Emotions Scale: Validity, reliability and sensitivity to change. Journal of Psychosomatic Research, 2010. 68(3): p. 285-292.

25. Ali, A., et al., Emotional abuse, self-blame, and self-silencing in women with irritable bowel syndrome. Psychosom Med, 2000. 62(1): p. 76-82.

26. Bowers, H. and A. Wroe, Beliefs about emotions mediate the relationship between emotional suppression and quality of life in irritable bowel syndrome. J Ment Health, 2016. 25(2): p. 154-8.

27. Bowers, H., A. Wroe, and T. Pincus, The relationship between beliefs about emotions and quality of life in irritable bowel syndrome. Psychol Health Med, 2017: p. 1-7.

28. Halpert, A., D. Rybin, and G. Doros, Expressive Writing Is a Promising Therapeutic Modality for the Management of IBS: A Pilot Study. American Journal of Gastroenterology, 2010. 105(11): p. 2440-2448.

29. Bowers, H., A.L. Wroe, and T. Pincus, 'Isn't it ironic?' Beliefs about the unacceptability of emotions and emotional suppression relate to worse outcomes in fibromyalgia. Clinical Rheumatology, 2017. 36(5): p. 1121-1128.

30. Campbell-Sills, L., et al., Acceptability and suppression of negative emotion in anxiety and mood disorders. Emotion, 2006. 6(4): p. 587-595.

31. Pressman, S.D. and S. Cohen, Does positive affect influence health? Psychol Bull, 2005. 131(6): p. 925-971.

32. Muscatello, M.R., et al., Personality traits and emotional patterns in irritable bowel syndrome. World J Gastroenterol, 2016. 22(28): p. 6402-15.

33. Ben-Ezra, M., et al., Cognitive Appraisal and Psychological Distress Among Patients with Irritable Bowel Syndrome. Israel Journal of Psychiatry and Related Sciences, 2015. 52(1): p. 54-60.

34. Charlson, M.E., et al., Mediators and moderators of behavior change in patients with chronic cardiopulmonary disease: The impact of positive affect and self-affirmation. Translational Behavioral Medicine, 2014. 4(1): p. 7-17.

35. Finan, P.H. and E.L. Garland, The Role of Positive Affect in Pain and Its Treatment. Clinical Journal of Pain, 2015. 31(2): p. 177-187.

36. DeSteno, D., J.J. Gross, and L. Kubzansky, Affective Science and Health: The Importance of Emotion and Emotion Regulation. Health Psychology, 2013. 32(5): p. 474-486.

37. Everitt, H., et al., Assessing Cognitive behavioural Therapy in Irritable Bowel (ACTIB): protocol for a randomised controlled trial of clinical-effectiveness and cost-effectiveness of therapist delivered cognitive behavioural therapy and web-based self-management in irritable bowel syndrome in adults. BMJ Open, 2015. 5(7): p. e008622.

38. Francis, C.Y., J. Morris, and P.J. Whorwell, The irritable bowel severity scoring system: $a$ simple method of monitoring irritable bowel syndrome and its progress. Aliment Pharmacol Ther, 1997. 11(2): p. 395-402.

39. Zigmond, A.S. and R.P. Snaith, The hospital anxiety and depression scale. Acta Psychiatr Scand, 1983. 67(6): p. 361-70.

40. Muthén, L.K. and B.O. Muthén, Mplus User's Guide. Seventh Edition ed. 1998-2015, Los Angeles, CA: Muthén \& Muthén.

41. Norton, S., et al., The Hospital Anxiety and Depression Scale: a meta confirmatory factor analysis. J Psychosom Res, 2013. 74(1): p. 74-81.

42. Luciano, J.V., et al., Bifactor Analysis and Construct Validity of the HADS: A Cross-Sectional and Longitudinal Study in Fibromyalgia Patients. Psychological Assessment, 2014. 26(2): p. 395-406.

43. Watson, D., L.A. Clark, and A. Tellegen, Development and validation of brief measures of positive and negative affect: the PANAS scales. J Pers Soc Psychol, 1988. 54(6): p. 1063-70. 
44. Mundt, J.C., et al., The Work and Social Adjustment Scale: a simple measure of impairment in functioning. Br J Psychiatry, 2002. 180: p. 461-4.

45. Organization, W.H., International Classification of Functioning, Disability and Health: ICF, 2001, World Health Organization: Geneva.

46. IBM, IBM SPSS Statistics for Windows, Version 21.0, 2012, IBM Corp: Armonk, NY.

47. Mackinnon, D.P., C.M. Lockwood, and J. Williams, Confidence Limits for the Indirect Effect: Distribution of the Product and Resampling Methods. Multivariate Behav Res, 2004. 39(1): p. 99.

48. Hu, L.T. and P.M. Bentler, Cutoff Criteria for Fit Indexes in Covariance Structure Analysis: Conventional Criteria Versus New Alternatives. Structural Equation Modeling-a Multidisciplinary Journal, 1999. 6(1): p. 1-55.

49. Dion, K.L., Ethnolinguistic correlates of alexithymia: toward a cultural perspective. J Psychosom Res, 1996. 41(6): p. 531-9.

50. Le, H.N., H. Berenbaum, and C. Raghavan, Culture and alexithymia: mean levels, correlates, and the role of parental socialization of emotions. Emotion, 2002. 2(4): p. 341-60.

51. Lumley, M.A., et al., Alexithymia and pain in three chronic pain samples: comparing Caucasians and African Americans. Pain Med, 2005. 6(3): p. 251-61.

52. Gross, J.J. and R.W. Levenson, Emotional suppression: physiology, self-report, and expressive behavior. J Pers Soc Psychol, 1993. 64(6): p. 970-86.

53. Gross, J.J. and R.W. Levenson, Hiding feelings: the acute effects of inhibiting negative and positive emotion. J Abnorm Psychol, 1997. 106(1): p. 95-103.

54. Lam, S., et al., Emotion regulation and cortisol reactivity to a social-evaluative speech task. Psychoneuroendocrinology, 2009. 34(9): p. 1355-1362.

55. Bennett, E.J., et al., Suppression of Anger and Gastric-Emptying in Patients with Functional Dyspepsia. Scandinavian Journal of Gastroenterology, 1992. 27(10): p. 869-874.

56. Appleton, A.A., et al., Divergent Associations of Adaptive and Maladaptive Emotion Regulation Strategies With Inflammation. Health Psychology, 2013. 32(7): p. 748-756.

57. Mayer, E., The Mind-Gut Connect. 2016, New York: Harper Wave, Harper Collins.

58. Fredrickson, B.L., What Good Are Positive Emotions? Rev Gen Psychol, 1998. 2(3): p. 300319.

59. Corstorphine, E., Cognitive-emotional-behavioural therapy for the eating disorders: Working with beliefs about emotions. European Eating Disorders Review, 2006. 14(6): p. 448-461.

60. Rimes, K.A. and J. Wingrove, Mindfulness-based cognitive therapy for people with chronic fatigue syndrome still experiencing excessive fatigue after cognitive behaviour therapy: a pilot randomized study. Clin Psychol Psychother, 2013. 20(2): p. 107-17.

61. Porcelli, P., et al., Alexithymia as predictor of treatment outcome in patients with functional gastrointestinal disorders. Psychosom Med, 2003. 65(5): p. 911-8.

62. Moss-Morris, R., et al., A randomized controlled trial of a cognitive behavioural therapybased self-management intervention for irritable bowel syndrome in primary care. Psychol Med, 2010. 40(1): p. 85-94.

63. Kennedy, T., et al., Cognitive behaviour therapy in addition to antispasmodic treatment for irritable bowel syndrome in primary care: randomised controlled trial. BMJ, 2005. 331(7514): p. 435.

64. Lackner, J.M., et al., How does cognitive behavior therapy for irritable bowel syndrome work? A mediational analysis of a randomized clinical trial. Gastroenterology, 2007. 133(2): p. 433444.

65. Greenberg, L.S., Emotion-focused therapy of depression. Person Centered \& Experiential Psychotherapies, 2017. 16(2): p. 106-117.

66. Pressman, S.D. and S. Cohen, Does Positive Affect Influence Health? Psychological Bulletin, 2005. 131(6): p. 925-971. 
67. Algoe, S.B. and A.L. Stanton, Gratitude When It Is Needed Most: Social Functions of Gratitude in Women With Metastatic Breast Cancer. Emotion, 2012. 12(1): p. 163-168.

68. Fredrickson, B.L., The role of positive emotions in positive psychology. The broaden-and-build theory of positive emotions. Am Psychol, 2001. 56(3): p. 218-26.

69. Finan, P.H.P. and E.L.P. Garland, The Role of Positive Affect in Pain and Its Treatment. Clinical Journal of Pain, 2015. 31(2): p. 177-187.

70. Whorwell, P.J., et al., Physiological-Effects of Emotion - Assessment Via Hypnosis. Lancet, 1992. 340(8811): p. 69-72.

71. Richards, J.M. and J.J. Gross, Emotion regulation and memory: The cognitive costs of keeping one's cool. Journal of Personality and Social Psychology, 2000. 79(3): p. 410-424.

72. Gross, J.J., Handbook of emotion regulation. 2007, New York, NY: Guilford Press.

73. Deary, V., T. Chalder, and M. Sharpe, The cognitive behavioural model of medically unexplained symptoms: a theoretical and empirical review. Clin Psychol Rev, 2007. 27(7): p. 781-97.

74. Hauser, G., S. Pletikosic, and M. Tkalcic, Cognitive behavioral approach to understanding irritable bowel syndrome. World J Gastroenterol, 2014. 20(22): p. 6744-58.

75. Spence, M.J. and R. Moss-Morris, The cognitive behavioural model of irritable bowel syndrome: a prospective investigation of patients with gastroenteritis. Gut, 2007. 56(8): p. 1066-1071.

76. Sibelli, A., et al., The role of high expectations of self and social desirability in emotional processing in individuals with irritable bowel syndrome: A qualitative study. $\mathrm{Br} \mathrm{J}$ Health Psychol, 2017.

77. Hakanson, C., Everyday Life, Healthcare, and Self-Care Management Among People With Irritable Bowel Syndrome An Integrative Review of Qualitative Research. Gastroenterology Nursing, 2014. 37(3): p. 217-225.

78. Koole, S., The psychology of emotion regulation: An integrative review. Cognition \& Emotion, 2009. 23(1): p. 4-41.

79. Aldao, A., S. Nolen-Hoeksema, and S. Schweizer, Emotion-regulation strategies across psychopathology: A meta-analytic review. Clin Psychol Rev, 2010. 30(2): p. 217-37. 